\title{
Klosterfrau-Preise nach Bern und München
}

Im Rahmen der 31. Jahrestagung der Deutschen Gesellschaft für Pädiatrische Pneumologie in Düsseldorf wurde am 28. März der Berner Pädiater Dr. Philipp Latzin mit dem „Klosterfrau Forschungspreis 2009" ausgezeichnet. Die Münchner Kinderärztin Dr. Bianca Schaub erhielt den „Klosterfrau Research Grant 2009“.

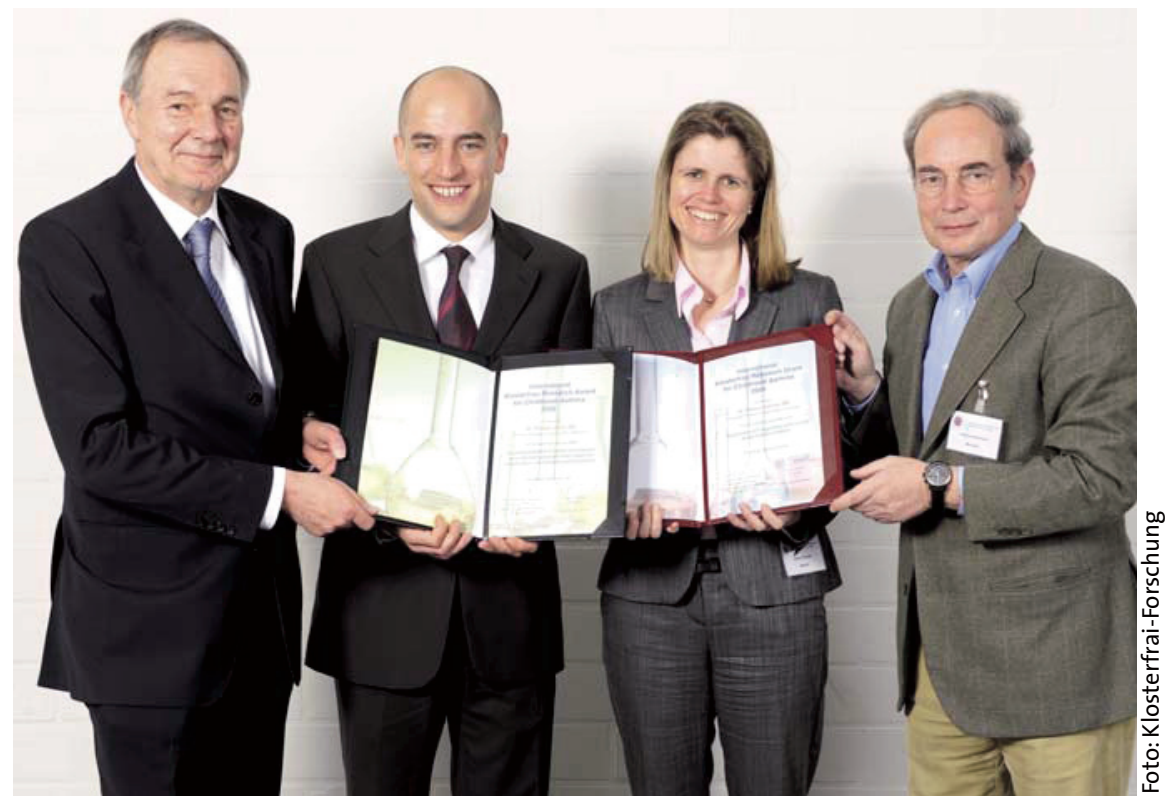

Von links nach rechts: Dr. Harald Greve, Leitung Wissenschaft und Medizin bei Klosterfrau, die Preisträger Dr. Philipp Latzin und Dr. Bianca Schaub, Prof. Dr. Dietrich Reinhardt, Vorsitzender der internationalen Expertenjury

D r. Philipp Tobias Latzin promovierte nach seinem Studium an der Ludwig-Maximilians-Universität, München, am Dr. von Haunerschen Kinderspital in der Forschungsgruppe von Prof. Dr. Matthias Griese von 1997 bis 1999. Dort arbeitete der angehende Facharzt für Kinderheilkunde nach verschiedenen Praktika von 2002 bis 2004 erneut. Seit 2005 ist er am Kinderhospital der Universitätsklinik in Bern tätig.

Latzin gelang erstmals der Nachweis, dass die pränatale Exposition gegenüber Feinstaub und auch gegenüber dem aus Autoabgasen stammenden Stickstoffdioxid mit einer eingeschränkten Lungenfunktion bei Neugeborenen einhergeht. Zudem fand er bei Kindern von atopischen Müttern einen Zusammenhang zwischen der erhöhten
Exhalation von Stickstoffmonoxid (eNO) kurz nach der Geburt und schweren respiratorischen Störungen im ersten Lebensjahr. Zwar etwas schwächer ausgeprägt, aber ebenfalls eindeutig war dieser Zusammenhang bei Kindern von Müttern, die während der Schwangerschaft geraucht hatten. Die frühe, nicht invasive Messung von eNO könnte damit eventuell Säuglinge mit einem sehr hohen Risiko für allergisches Asthma identifizieren. Einen wesentlichen Beitrag leistete Latzin auch zur Diagnose der allergischen bronchopulmonalen Aspergillose. Er entdeckte, dass das Chemokin TARC („thymus and activation regulated chemokine") bei dieser Erkrankung erhöht und allen anderen diagnostischen Markern überlegen ist.
Dr. Bianca Schaub studierte Medizin in München und Dundee, Schottland. Nach der Promotion ging sie von 2002 bis 2004 als PhD nach Boston. Seit 2004 leitet sie das Labor für Allergie und Immunologie an der Universitätskinderklinik München und bereitet sich auf ihre Habilitation vor.

Entscheidend für die Entwicklung allergischer Erkrankungen bei Kindern sind frühe Immunmechanismen, die insbesondere durch regulatorische $\mathrm{T}$ Zellen vermittelt werden. $\mathrm{Zu}$ diesem Ergebnis kommt Schaub in ihren Forschungsarbeiten. Ein solches tiefergehendes Verständnis für diese Vorgänge der Entwicklung und Reifung des Immunsystems trägt entscheidend zur Entwicklung präventiver Strategien bei.

Der internationale Klosterfrau Forschungspreis ist mit $30.000 €$ dotiert und zeichnet jedes Jahr Wissenschaftler aus, die sich in der Grundlagenforschung, Diagnostik und Therapie von kindlichem Asthma bronchiale verdient machen. Der Klosterfrau Research Grant ist mit $20.000 €$ dotiert und würdigt wissenschaftliche Verdienste in der Grundlagenforschung.

red

Kanert-Preis für Allergieforschung Die Stiftung Kanert für Allergieforschung schreibt für 2009 wieder den „KanertPreis für Allergieforschung" aus. Der Preis wird nicht für eine bisherige Forschungsleistung vergeben, sondern soll ein künftiges Projekt unterstützen. Der Preis ist mit einer Summe von $\mathbf{1 0 . 0 0 0}$ Euro dotiert.

Anträge sollen in Form einer Projektskizze beim Kuratorium der Stiftung eingereicht werden. Aus der Skizze sollten Stand der Forschung, die eigenen Vorarbeiten, die eingesetzten Methoden und die Ziele hervorgehen. Außerdem ist ein Lebenslauf beizufügen. Abgabefrist ist der 15. Juli 2009.

\section{Prof. Dr. Dr. Johannes Ring}

Klinik und Poliklinik für Dermatologie und Allergologie am Biederstein Biedersteiner Straße 29

80802 München

Tel.: (o 89) 41 40-31 70/-32 17

Fax: (o 89) 41 40-31 71

E-Mail: johannes.ring@Irz.tum.de 